\title{
Evaluación del uso de señales visuales y de localización por el colibrí cola-ancha (Selasphorus platycercus) al visitar flores de Penstemon roseus
}

\section{Evaluation of the use of visual and location cues by the Broad-tailed hummingbird (Selasphorus platycercus)foraging in flowers of Penstemon roseus}

\author{
Guillermo Pérez ${ }^{1 \bowtie}$, Carlos Lara $^{2}$, Martha Signoret-Poillon ${ }^{3}$ y José A. Viccon-Pale ${ }^{3}$ \\ ${ }^{1}$ Universidad Autónoma Metropolitana. Unidad Xochimilco. Calzada del Hueso 1100, Col. Villa Quietud 04960 México D.F., México. \\ ${ }^{2}$ Laboratorio de Ecología de la Conducta, Centro Tlaxcala de Biología de la Conducta, Universidad Autónoma de Tlaxcala. Carretera Tlaxcala- \\ Puebla. Km 1.5 s/n, 90070 Tlaxcala, Tlaxcala, México. \\ ${ }^{3}$ Departamento El Hombre y su Ambiente, Universidad Autónoma Metropolitana, Unidad Xochimilco. Calzada del Hueso 1100, Col. Villa Quietud \\ 04960 México D.F., México. \\ \gaperezf@hotmail.com
}

\begin{abstract}
Resumen. En los colibríes la memoria espacial desempeña un papel importante durante el forrajeo. Éste se basa en el uso de señales específicas (visuales) o en señales espaciales (localización de flores y plantas con néctar). Sin embargo, el uso de estas señales por los colibríes puede variar de acuerdo con la escala espacial que enfrentan cuando visitan flores de una o más plantas durante el forrajeo; ésto se puso a prueba con individuos del colibrí cola-ancha Selasphorus platycercus. Por otro lado, para evaluar la posible variación en el uso de las señales, se llevaron a cabo experimentos en condiciones semi-naturales utilizando flores de la planta Penstemon roseus, nativa del sitio de estudio. A través de la manipulación de la presencia/ausencia de una recompensa (néctar) y señales visuales, evaluamos el uso de la memoria espacial durante el forrajeo entre 2 plantas (experimento 1) y dentro de una sola planta (experimento 2). Los resultados demostraron que los colibríes utilizaron la memoria de localización de la planta de cuyas flores obtuvieron recompensa, independientemente de la presencia de señales visuales. Por el contrario, en flores individuales de una sola planta, después de un corto periodo de aprendizaje los colibríes pueden utilizar las señales visuales para guiar su forrajeo y discriminar las flores sin recompensa. Asimismo, en ausencia de señales visuales los individuos basaron su forrajeo en la memoria de localización de la flor con recompensa visitada previamente. Estos resultados sugieren plasticidad en el comportamiento de forrajeo de los colibríes influenciada por la escala espacial y por la información adquirida en visitas previas.
\end{abstract}

Palabras clave: memoria espacial, señales visuales, localización, escala espacial.

\begin{abstract}
In hummingbirds spatial memory plays an important role during foraging. It is based in use of specific cues (visual) or spatial cues (location of flowers and plants with nectar). However, use of these cues by hummingbirds may change according to the spatial scale they face when visit flowers of one or more plants during foraging. This was tested with individuals of broad-tailed Hummingbird Selasphorus platycercus. To assess the possible variation in the use of cues, experiments were conducted in semi-natural conditions using flowers of Penstemon roseus plant, native in study site. By manipulating the presence/absence of a reward (nectar) and visual cues, we evaluated the use of spatial memory during foraging between 2 plants (experiment 1) and within a single plant (experiment 2). The results showed that hummingbirds used memory of location of plant in whose flowers obtained reward, regardless of the presence of visual cues. On the contrary, in individual flowers of a single plant, after a short learning period hummingbirds can use visual cues to guide their foraging and discriminate the flowers without reward. Also, in the absence of visual cues individuals based their foraging on memory of location of rewarded flower previously visited. These results suggest plasticity in the foraging behavior of hummingbirds influenced by the spatial scale and acquired information from previous visits.
\end{abstract}

Key words: spatial memory, visual cues, location, spatial scale. 


\section{Introducción}

Los colibríes dependen casi exclusivamente del néctar obtenido de las flores para su alimentación, por ello tienden a defender activamente territorios conformados por plantas que puedan proveerlo satisfactoriamente, y/o a desplazarse a distintas distancias estableciendo rutas de forrajeo que incluyen la visita a flores de muchas plantas (Feinsinger y Colwell, 1978; Garrison y Gass, 1999). Las flores que visitan constituyen una enorme diversidad de formas y colores, y el patrón de producción del néctar ofrecido es igualmente variable (Healy y Hurly, 2003; Lara, 2006). Adicionalmente, durante sus movimientos locales o migratorios, los colibríes pueden enfrentar variaciones espaciales y temporales en la disponibilidad de sus recursos florales (Gass et al., 1999). Por todo ello, al forrajear en la naturaleza, un colibrí debe tomar decisiones sobre qué parches, plantas o flores visitar inicialmente, en función de la recompensa de néctar (Pyke, 1978a; Gass y Montgomerie, 1981) y, por otro lado, usar la memoria para evitar volver a visitar las flores que ha vaciado recientemente (Cole et al., 1982; Gass y Sutherland, 1985; Brown y Gass, 1993; Brown, 1994; Hurly, 1996).

El uso de la memoria espacial ha sido documentado en colibríes forrajeando en flores artificiales y bebederos, tanto en condiciones de laboratorio como de campo, sugiriéndose que esta aves pueden recordar características visuales de las flores que visitan y su ubicación particular en el arreglo (Healy y Hurly, 2001). En este sentido, algunos estudios definen el uso de señales visuales en estos contextos como las asociaciones aprendidas de las características visibles en las flores (forma o color) y la cantidad o concentración de néctar que contienen (Bené, 1945; Miller y Miller, 1971; Stiles, 1976; George, 1980; Cole et al., 1982; Miller et al., 1985; Brown y Gass, 1993; Brown, 1994; Hurly y Healy, 1996, 2002; Melendez-Ackerman et al., 1997; Irwin, 2000). También hay evidencia que sugiere que los colibríes pueden atender a señales espaciales cuando realizan sus visitas y regresar a flores específicas, basándose para su localización en la memoria (Wolf y Hainsworth, 1990; Sutherland y Gass, 1995; Healy y Hurly, 1995,1998; Hurly, 1996; Hurly y Healy, 1996, 2002; Henderson et al., 2001; GonzálezGómez y Vázquez, 2006).

No obstante la evidencia antes mencionada, resulta interesante que la mayor parte de la información generada en Norteamérica, con respecto al uso de la memoria espacial en colibríes, haya sido obtenida a partir de especies que realizan movimientos migratorios (véase González-Gómez y Vázquez, 2006) y con frecuencia utilizando flores y arreglos artificiales (Cole et al., 1982; Grant y Temeles, 1992; Brown y Gass, 1993; Brown, 1994; Healy y Hurly, 1995, 1998; Sutherland y Gass, 1995; Hurly y Healy, 1996, 2002; Meléndez-Ackerman et al., 1997; Henderson et al., 2001).
Si consideramos que los colibríes son el segundo grupo de aves más diverso en el mundo (con más de 360 especies), distribuidas a lo largo de todo el continente americano, y que dependen de las flores de más de 100 especies de angiospermas, resulta evidente la necesidad de estudiar estas habilidades cognitivas no sólo en un mayor número de especies de colibríes incluyendo las residentes, sino además tomando en consideración las flores naturales en las evaluaciones.

Adicionalmente, es también interesante que la memoria espacial de estas aves haya sido evaluada registrando las visitas en arreglos o bebederos artificiales individuales, dado que en condiciones naturales los colibríes pueden enfrentar parches de flores conformados por más de una planta, y donde el uso de señales visuales y de localización puede ser distinto.

Aun cuando en condiciones experimentales el uso de plantas resulta difícil, dada la variabilidad en tamaño y forma de los individuos, el uso de flores naturales (incluso en arreglos artificiales) para evaluar la memoria espacial en colibríes puede ofrecer una aproximación más certera que la que se ha obtenido con dispositivos artificiales y que requiere de entrenamiento previo de los individuos. Por ello, resulta importante conservar en la medida de lo posible, el contexto ecológico en el que se desarrollan los colibríes para obtener datos más reales.

En el presente estudio, se realizaron experimentos de campo con una población de colibríes Selasphorus platycercus (colibrí cola ancha) residente en La Malinche, Tlaxcala; se evaluó el uso de la memoria de localización y de señales visuales durante su forrajeo en la planta Penstemon roseus (Plantaginaceae); el estudio se enfocó en evaluar estas habilidades cognitivas cuando los individuos forrajean en 2 plantas (Experimento 1) y cuando forrajean en flores de una planta (Experimento 2).

\section{Materiales y métodos}

El trabajo de campo se realizó de junio a noviembre de 2008 en el Parque Nacional La Malinche, Tlaxcala, México (19 $14^{\prime}$ N, 98 $8^{\circ} 58^{\prime}$ O, 3000 m snm). Selasphorus platycercus es una especie con poblaciones migratorias y residentes (Williamson, 2001). El sitio de estudio es uno de los lugares donde es posible localizar poblaciones residentes a lo largo de todo el año, aunque su abundancia es mayor entre octubre y enero (Lara, 2006). Este colibrí es visitante frecuente de parches de la planta Penstemon roseus (Cerv. ex Sweet) G. Don, una especie con flores tubulares hermafroditas de color magenta, y con un periodo de floración que se extiende de julio a diciembre, sus flores producen $6.5 \pm 0.45 \mu$ de néctar y $0.24 \pm 0.007 \mathrm{mg} / \mathrm{ml}$ de azúcar después de $24 \mathrm{~h}$ de acumulación (Lara, 2006; Lara y Ornelas, 2008; Lara et al., 2009). 
Se capturaron 19 colibríes adultos con redes de niebla, los cuales se sometieron a los experimentos de manera individual. Los experimentos se realizaron sistemáticamente de las 800-1 500 horas. Las aves se mantuvieron individualmente en jaulas de $1 \times 1 \times 1 \mathrm{~m}$, desarmables, y provistas con una percha de madera. Las jaulas fueron colocadas en la proximidad del sitio de captura en las condiciones ambientales de luz y temperatura del sitio. Previo a las pruebas experimentales, se permitió que cada individuo se aclimatara en la jaula por 10-15 min durante los cuales no se alimentaron. Se consideró señal de aclimatación cuando el colibrí se posó sobre la percha y entonces se inició cada experimento.

Para el experimento 1 se emplearon 50 plantas de la especie Penstemon roseus con características de tamaño $(\sim 1 \mathrm{~m})$ y floración (al menos 10 flores abiertas) similares. Las plantas se extrajeron del suelo, se trasplantaron a bolsas de polietileno y se mantuvieron en las condiciones ambientales naturales del sitio de estudio, 30 días antes de iniciarse los experimentos. Para el experimento 2 se utilizaron flores colectadas de las plantas antes mencionadas.

Experimento 1. Visitas a plantas. Localización o señales visuales. En este experimento los sujetos de estudio fueron 6 colibríes ( 3 hembras y 3 machos). Para determinar si los colibríes utilizan memoria de localización o señales visuales para regresar a flores previamente visitadas cuando forrajean en más de una planta, este experimento consistió en 2 fases:

Fase 1 (de entrenamiento). Se colocaron 2 plantas dentro de la jaula, con una distancia de separación de $80 \mathrm{~cm}$ entre plantas. Para controlar el número de flores ofrecidas, cada una de las plantas tuvo 4 flores abiertas (las restantes fueron desprendidas manualmente), a las cuales se les extrajo todo el néctar usando tubos capilares. Antes de cada prueba experimental, las 4 flores de 1 de las 2 plantas se llenaron con $100 \mu \mathrm{l}$ de solución de sacarosa al $20 \%$ (en peso), (néctar o recompensa) y se marcaron en la base de la corola con etiquetas de color amarillo brillante $(1 \times 2 \mathrm{~cm})$ a manera de señal visual. Las 4 flores de la otra planta no se marcaron ni se les agregó solución de sacarosa y se les designó flores distractoras o flores irrelevantes. Para cada prueba experimental, se introdujo un colibrí a la jaula y se registraron sus visitas por 15 minutos. Para cada individuo se consideró como 1), el número de flores visitadas en ambas plantas y como 2), el tiempo (minutos y segundos) en que el colibrí visitó cada flor. Al final de la primera fase el colibrí evaluado se extrajo sin manipulación directa (colocando al lado otra jaula vacía a la que se transladó durante 5 minutos), antes de iniciar la siguiente fase. La cantidad de néctar utilizado en este experimento, tuvo la finalidad de mantener el estímulo durante el tiempo de la fase de entrenamiento.
Fase 2 (de retorno). Se disociaron las señales visuales de la localización previa de la recompensa; para ello, ambas plantas permanecieron en el mismo lugar dentro de la jaula, pero se extrajo el remanente de solución de sacarosa de las flores y las etiquetas se colocaron en las flores de la otra planta; después nuevamente se introdujo al colibrí en la jaula y se registraron sus visitas por 5 minutos más, siguiendo el protocolo arriba descrito.

Una vez completas las 2 fases, los colibríes evaluados se marcaron con un corte en la punta de la quinta pluma rectriz (para reconocerlos en caso de recaptura) y se liberaron.

Experimento 2. Visitas a flores en una planta. La habilidad de memorizar señales visuales y localizaciones de flores específicas cuando los colibríes visitaban una sola planta, fue evaluada a través de 2 tratamientos distintos. Sin embargo, para ambos tratamientos fue necesario eliminar la posible variación de las plantas utilizadas, con respecto al tamaño, vigor (apariencia saludable de la planta) o incluso el ángulo de inclinación. De esta manera, se decidió usar estacas verticales de madera de color verde (de $50 \mathrm{~cm}$ de longitud). En estas estacas fueron insertadas 4 flores de $P$. roseus sobre receptáculos individuales de agujas de jeringa. La distancia entre flores fue de $5 \mathrm{~cm}$, y la primera se colocó a $20 \mathrm{~cm}$ del suelo. La estaca se clavó en el centro de la jaula experimental.

Tratamiento 1. Memoria de señales visuales. Con el fin de determinar la capacidad de los colibríes para asociar la recompensa de néctar con las señales visuales y utilizarlas posteriormente para guiar su forrajeo, una de las 4 flores insertadas en la estaca se llenó con $300 \mu \mathrm{l}$ de solución de sacarosa al $20 \%$ para mantener un remanente de néctar en la flor recompensada que estimulara el regreso. Esta flor se marcó en la base de la corola con la misma señal visual descrita en el experimento 1. Las 3 flores restantes estuvieron vacías y sin marca (flores irrelevantes). En todos los experimentos la posición de la flor marcada y recompensada se asignó al azar.

Para este tratamiento se utilizaron 6 colibríes ( 5 machos y 1 hembra). Cada individuo se sometió a 4 pruebas. En una prueba, se introdujo un colibrí a la jaula provista de una estaca con flores y durante 5 minutos se registró el número de visitas que les hizo. Después de este tiempo, la estaca se retiró y se cambió de posición la flor marcada. El néctar remanente en esa flor se vació y nuevamente se rellenó con $300 \mu \mathrm{l}$ para mantener el estímulo de recompensa asociado con la señal visual. Pasados 5 minutos, el arreglo se introdujo en la jaula y se repitió el protocolo de registro. Este método se repitió hasta completar 4 pruebas por individuo, después de lo cual el colibrí se marcó y se liberó.

Tratamiento 2. Memoria de localización. Para evaluar el uso de la memoria de localización por $S$. platycercus al guiar su forrajeo en flores individuales, se utilizaron 7 individuos 
diferentes (4 hembras y 3 machos). Las estacas fueron idénticas a las del tratamiento 1, pero la flor con néctar no estuvo marcada y su posición fue constante; sin embargo, las flores se renovaron en las 4 pruebas para evitar que los rasgos de la flor con recompensa pudieran utilizarse como señal visual. El protocolo de rellenado de néctar entre pruebas, registro de visitas, marcaje y liberación de los colibríes evaluados fue el mismo que para el tratamiento 1.

Análisis de datos. En ambos experimentos, la variación en el porcentaje de visitas en la localización correcta o en las señales visuales se evaluó utilizando análisis de varianza de medidas repetidas (Zar, 1999). En el modelo, el tipo de flor visitada (localización, señal visual o flor irrelevante) fue tratado como factor fijo y el porcentaje de visitas en la fase 2 (experimento 1) o pruebas (experimento 2) fueron las medidas repetidas. Los datos se transformaron al arcoseno antes del análisis estadístico para establecer normalidad.

Para determinar el uso de la memoria de localización o de la señal visual en las primeras elecciones, se utilizó un análisis de chi-cuadrada. Se evaluaron los datos combinados de las 3 primeras visitas en el experimento 1 (fase 2) y la primera visita en las pruebas 2, 3 y 4 para el experimento 2 (ambos tratamientos) contra un modelo nulo, preferencias al azar (Zar, 1999).

Se utilizaron análisis de supervivencia, para estimar la probabilidad de visita de los colibríes en ambas fases del experimento 1, y el método no paramétrico de producto límite de Kaplan-Meier para el cálculo de la probabilidad de que los colibríes no hayan visitado una flor en la planta 5 minutos después de iniciar la observación y la prueba de Logrank (Mantel-Cox) para probar diferencias entre los tipos de flores (Muenchow, 1986). Todos los análisis estadísticos se hicieron mediante el programa StatView 5.0 (SAS Institute, 1998).

\section{Resultados}

Visitas a plantas. Localización o señales visuales. Los datos obtenidos en la primera fase del experimento 1 (entrenamiento) mostraron que los colibríes realizaron más visitas a las flores con la señal visual y recompensa $(80.7 \% \pm 4.7)$, que a las flores irrelevantes $(19.3 \% \pm 4.7)$. De igual manera, en la segunda fase (de retorno), los individuos realizaron más visitas a las flores en la localización previa de la recompensa $(67.5 \% \pm 11.86)$ que a las flores con la señal visual (antes irrelevantes) $(32.5 \% \pm 11.86)$, $\left(F_{1,10}=15.94, p=0.002\right)$. Por ello, la intensidad de visita (el porcentaje total de visitas a las flores de las 2 plantas) en ambas fases fue similar $\left(F_{1,10}=0.03, p=0.857\right)$. Igualmente, al comparar el porcentaje de visitas hechas en cada tipo de planta (en la localización correcta y flores irrelevantes) entre fases, no se encontraron diferencias estadísticamente significativas $\left(F_{1,10}=1.15, p=0.307\right)$ (Fig. 1).

Durante la segunda fase, la mayoría de los colibríes realizaron las 3 primeras visitas a la planta en la localización previa del néctar y no en la planta con las señales visuales $\left(\chi_{0.05,1}^{2}=3.84, p<0.008\right)$. En la primera y segunda visitas, 5 de los 6 colibríes evaluados eligieron en su primera visita la planta con la localización previa del néctar. En la tercera visita, 4 colibríes visitaron la localización previa del néctar, uno visitó la planta con las señales visuales y un colibrí dejó de visitar las plantas y se perchó hasta el final de la fase 2 (Fig. 2). Las visitas 2 y 3 en la planta con señales visuales fueron hechas por el mismo colibrí.

Las curvas de probabilidad de visita de los colibríes en las 2 fases experimentales no mostraron diferencias significativas (Fig.3). En la fase 1, las flores con néctar marcadas con señales visuales tuvieron la misma probabilidad de ser visitadas por los colibríes que las flores irrelevantes (Logrank (Mantel-Cox): $\chi^{2}=2.71 . g l=1, p=0.099$ ). De la misma manera, en la fase 2 , cuando las señales visuales se cambiaron a la otra planta, no se encontraron diferencias en la probabilidad de visita a las flores de ambas plantas (Logrank (Mantel-Cox): $\chi^{2}=0.094, g l=1, p=0.759$ ).

Visitas a flores en una planta. Cuando los colibríes forrajearon en un arreglo donde sólo 1 de las flores contuvo néctar, estuvo marcada con una señal visual y en posición variable durante 4 pruebas (experimento 2, tratamiento1), la mayoría de las visitas las recibió esa flor $\left(F_{1,30}=17.28\right.$, $p=0.002)$. Este patrón de visita se repitió a lo largo de las pruebas, sin generar diferencias significativas entre pruebas $\left(F_{3,30}=0.401, p=0.753\right)$. Se encontraron diferencias significativas en la proporción de visitas en cada tipo de flor (con señal visual y flores irrelevantes) entre pruebas $\left(F_{3,30}=7.49, p=0.007\right.$; Fig. 4$)$. En todos los periodos entre pruebas se verificó remanente de néctar.

Por otro lado, la flor con la señal visual no fue inicialmente elegida en la primera visita de los colibríes en las pruebas 2,3 y $4\left(\chi_{0.05,2}^{2}=5.99, p=0.644\right)$. Este comportamiento sugiere que al inicio de cada prueba los colibríes buscaron recompensa en todas las flores del arreglo, pero subsecuentemente usaron la señal visual para dirigir sus visitas hacia la flor que la contenía (Fig. 5).

En el tratamiento 2, cuando sólo una flor contuvo recompensa en el arreglo, pero su posición fue la misma en pruebas subsecuentes, los colibríes visitaron con mayor frecuencia a las flores en la localización de la recompensa que a las flores irrelevantes $\left(F_{1,36}=11.91, p=0.004\right)$ y no hubo variación estadística de este patrón entre pruebas $\left(F_{3,36}=0.112, p=0.952\right)$. La proporción de visitas en cada tipo de flor por prueba presentó variación significativa $\left(F_{3,36}=4.223, p=0.011\right)$ (Fig. 4). Nuevamente, se con- 


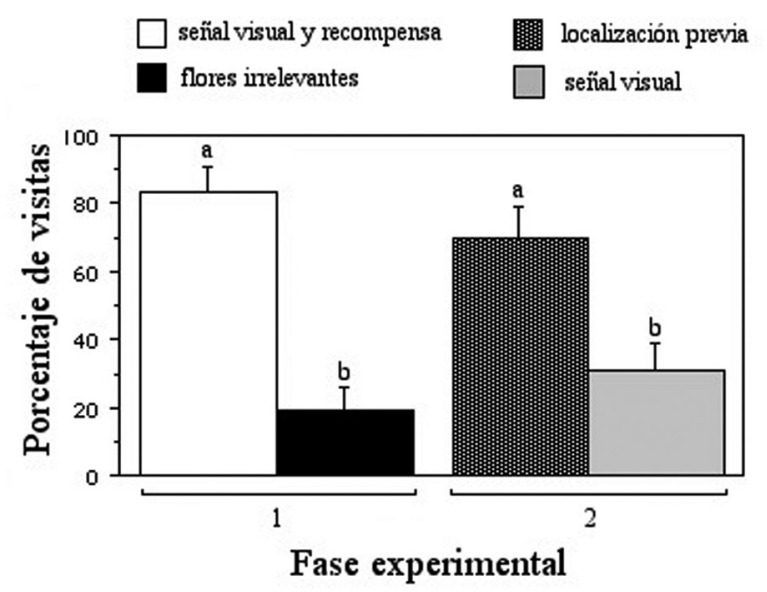

Figura 1. El porcentaje de visitas realizadas por 6 colibríes a cada tipo de flor en ambas fases del experimento 1. Los valores representan promedios y errores estándar (máximo $=100 \%$ ). Superíndices diferentes representan diferencias significativas entre grupos (ANDEVA de medidas repetidas; $p>0.05$ ).

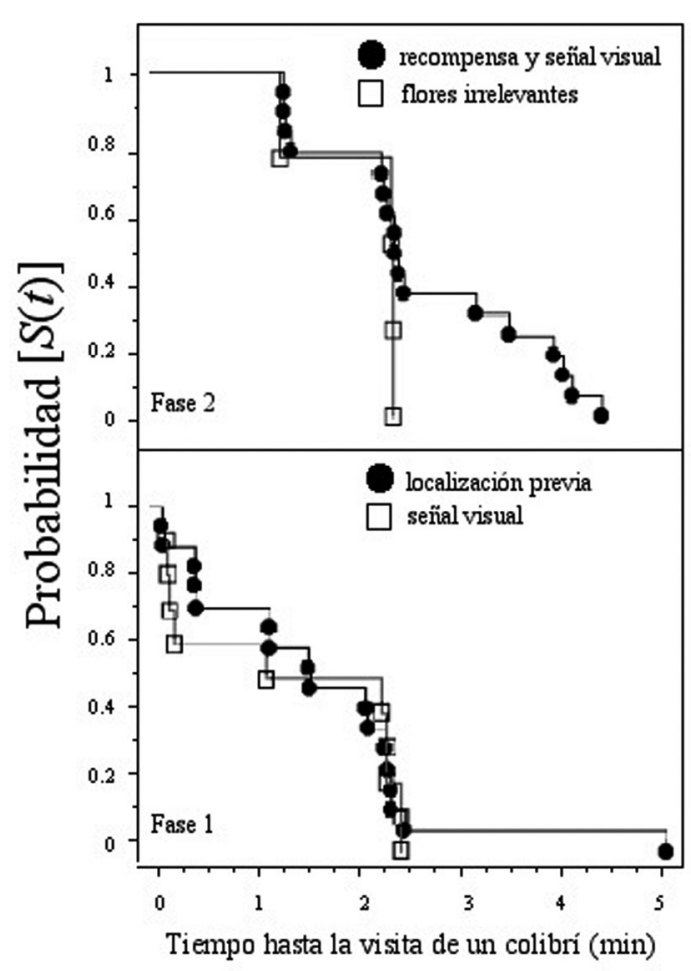

Figura 3. Comparación de la probabilidad de visita en la fase 1 (flores con señal visual con néctar y flores irrelevantes) y fase 2 (flores en localización previa sin néctar y flores con señal visual; experimento 1) durante un periodo de observación de $5 \mathrm{~min}$. La abreviatura $\mathrm{S}(\mathrm{t})$ en el eje $\mathrm{Y}$ es la probabilidad de que un colibrí aún no haya visitado una flor.

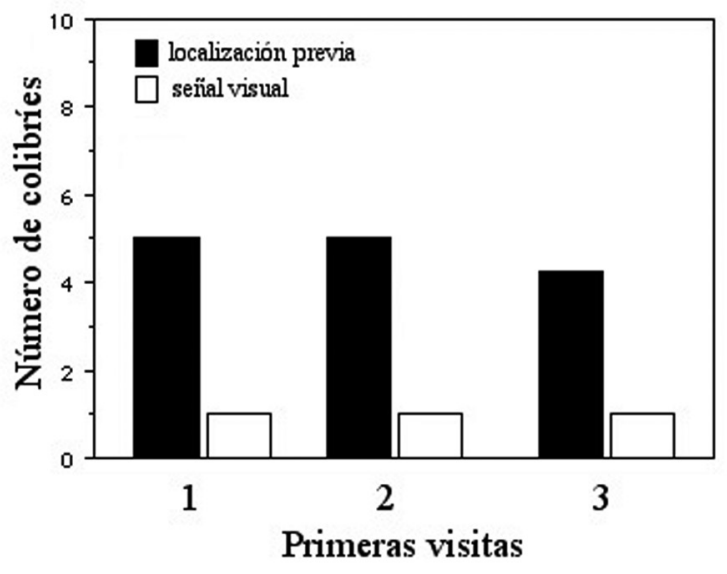

Figura 2. Las primeras 3 visitas realizadas por 6 colibríes a flores con señal visual (fase 1) y a flores ubicadas en la localización previa de la recompensa (fase 2) en el experimento 1.

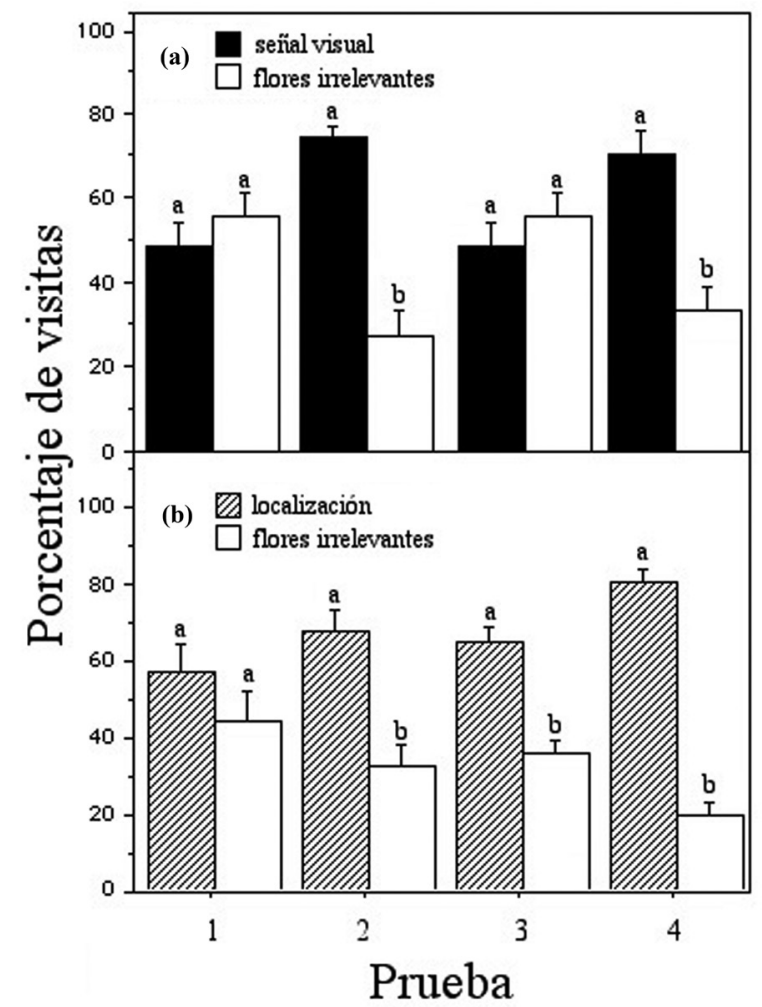

Figura 4. Porcentaje de visitas de los colibríes en los tratamientos 1 (señal visual [a]) y 2 (localización [b]) en 4 pruebas (exp. 2 ). Los valores corresponden a promedios \pm y errores estándar $($ máximo $=100 \%)$. Superíndices diferentes indican diferencias (ANDEVA de medidas repetidas; $p>0.05$ ). 


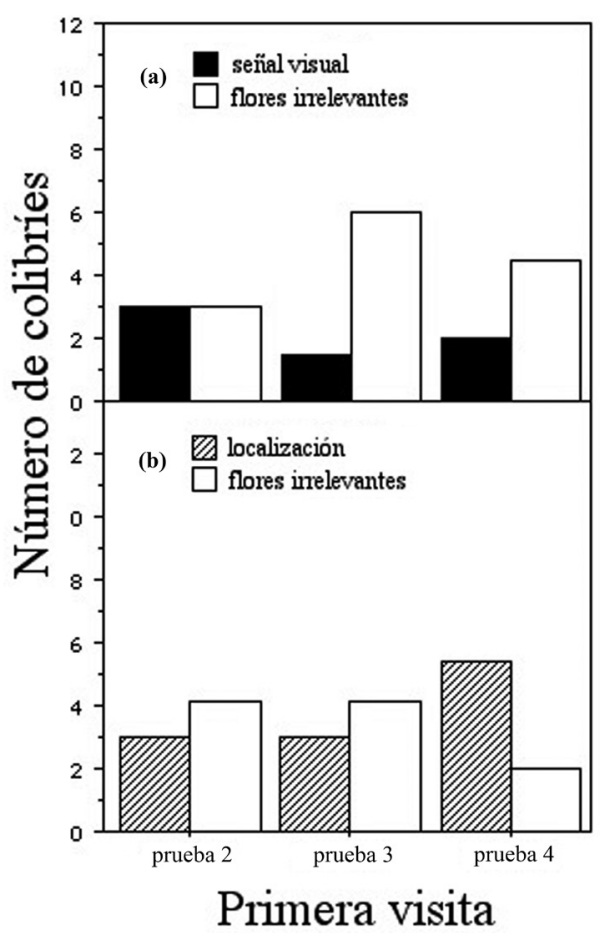

Figura 5. Primera visita registrada en cada prueba (exp.2) del tratamiento 1 (señal visual) y tratamiento 2 (localización).

firmó que la flor no fue vaciada en su totalidad en los 3 periodos entre pruebas.

No obstante, al analizar la primera visita de las pruebas 2, 3 y 4 no se encontraron diferencias en la cantidad de individuos que dirigieron su primera visita a la localización de la flor con recompensa y a flores irrelevantes $\left(\chi_{0.05,2}^{2}=5.99, p=0.509\right.$; Fig. 5). Los colibríes usaron un patrón de visitas similar al del tratamiento 1 antes de utilizar el recuerdo de la localización de la flor con recompensa.

\section{Discusión}

Los resultados obtenidos en este estudio muestran que los individuos de $S$. platycercus pueden utilizar la memoria de localización y las señales visuales, dependiendo de la escala espacial en la que el comportamiento de forrajeo se evalúe. De esta manera, los datos sugieren que cuando los colibríes forrajean entre 2 plantas, prefieren regresar a las plantas en las que previamente hallaron néctar de las cuales recuerdan su localización (experimento 1, fase 2); pero cuando forrajean en las flores individuales de una sola planta, pueden usar con eficiencia similar una señal visual o la memoria de localización para discernir entre flores con néctar y flores irrelevantes (experimento 2, tratamiento $1 \mathrm{y}$ tratamiento 2 ).
El hallazgo de que los colibríes pongan mayor atención a la localización que a las señales visuales cuando forrajean en 2 plantas (experimento 1) es un comportamiento demostrado previamente en otros experimentos de memoria espacial en laboratorio y en campo, especialmente con la especie Selasphorus rufus (Cole et al., 1982, Hurly y Healy, 1996; Healy y Hurly, 1998; Henderson et al., 2001; Healy y Hurly , 2003; véase también Sephanoides sephaniodes en González-Gómez y Vázquez, 2006). Dichos experimentos sugieren que la preferencia a responder señales espaciales por sobre señales visuales cuando los colibríes forrajean en más de una planta (arreglo artificial o bebedero), es probablemente la expresión de la señal que normalmente usan durante el forrajeo. La preferencia por regresar a sitios de alimento asociados a señales espaciales de localización también se ha observado en aves almacenadoras de alimento, como córvidos y páridos (Bossema, 1979; Balda y Kamil, 1989; Sherry, 1989), lo que revela que los colibríes pueden recordar información sobre la localización tan bien como el contenido del néctar en los sitios de forrajeo.

Los resultados obtenidos en el experimento 2 sugieren, por un lado, que los colibríes cuando forrajean en flores dentro de una sola planta se comportan como aves no almacenadoras y tienden a regresar frecuentemente a los sitios con la señal visual correcta (reforzada con una recompensa de néctar); por otro lado, tienden a recordar la localización de flores individuales. De esta manera, los colibríes pueden usar señales espaciales particulares (como la señal visual) o globales (como la localización), así como utilizar la información obtenida en visitas previas (Pyke, 1978a; Wolf y Hainsworth, 1990) y no el uso automático de un atrayente visual o reglas de decisión simples, como se ha descrito para abejas, abejorros y avispas (Pyke, 1978b; Corbet et al., 1981; Heinrich, 1983).

En este trabajo fue necesario considerar la cantidad del néctar como estímulo primario que desencadena el uso de las señales espaciales, en ambos experimentos se utilizó una cantidad excesiva (exp. $1400 \mu 1,100 \mu 1$ por flor; exp. $2300 \mu \mathrm{l}$ en una flor) para mantener el incentivo de retorno, que los colibríes asociaron a la señal visual o a la localización de la fuente de alimento. En un experimento similar con flores artificiales (Hurly y Healy, 1996) se utilizaron hasta $600 \mu \mathrm{l}$ para provocar el mismo efecto.

Tal como sugieren Hurly y Healy (1996), la preferencia en colibríes por atender o recordar la localización de las flores más que su color no parece sorprendente, debido a que los territorios de los colibríes contienen muchas flores de la misma especie. En consecuencia, sería ventajoso para un colibrí recordar qué flores de esa especie ha vaciado recientemente y evitarlas hasta que vuelvan a llenarse. Sin embargo, esto puede ser completamente diferente en 
ambientes más complejos, como los neotrópicos, donde numerosas especies de plantas florecen sincrónicamente, $\mathrm{y}$ es muy probable que un territorio contenga especies de plantas con flores de colores diferentes. Asimismo, las flores de una gran diversidad de plantas cambian de color con la edad (y sus volúmenes de néctar disminuyen). La presión selectiva en los colibríes para recordar localizaciones o señales visuales de muchas flores puede estar relacionada con las diferencias en las circunstancias ecológicas a las que se enfrentan.

En resumen, los resultados de ese estudio sugieren que los colibríes $S$. platycercus pueden atender o recordar señales espaciales globales cuando forrajean en más de una planta, pero pueden utilizar señales visuales estrechamente relacionadas con la flor cuando visitan una planta solitaria. Los datos del experimento 1 apoyan la afirmación de Hurly (1996) acerca de que los colibríes no sólo recuerdan la localización de las flores sino también sus contenidos, pero como se muestra en este estudio, eso puede depender de la escala espacial en la que su forrajeo sea evaluado.

\section{Agradecimientos}

A María Cristina Tello y Andrés Germán, por su apoyo en el trabajo de campo; al personal técnico de la Estación Científica La Malinche, Tlaxcala, por el acceso a las instalaciones y a los revisores por sus valiosos comentarios. Este trabajo fue apoyado por una beca del Consejo Nacional de Ciencia y Tecnología, México (Núm. 45914) otorgada a Guillermo Pérez.

\section{Literatura citada}

Balda, R. P. y A. C. Kamil. 1989. A comparative study of cache recovery by three corvid species. Animal Behaviour 38:486495.

Bené, F. 1945. The role of learning in the feeding behavior of black-chinned hummingbirds. Condor 47:3-21.

Bossema, I. 1979. Jays and oaks: an eco-ethological study of a symbiosis. Behaviour 70:1-117.

Brown, G. S. y C. L. Gass. 1993. Spatial association learning by hummingbirds. Animal Behaviour 46:487-497.

Brown, G. S. 1994. Spatial association learning by rufous hummingbirds (Selasphorus rufus): effects of relative spacing among stimuli. Journal of Comparative Psychology 108:29-35.

Cole, S., F .R. Hainsworth, A. C. Kamil, T. Mercier y L. L. Wolf. 1982. Spatial learning as an adaptation in hummingbirds. Science 217:655-657.

Corbet, S. A., I. Cutchill, M. Fallows, T. Harrison y G. Hartley. 1981. Why do nectar-foraging bees and wasps work upward on inflorescences? Oecologia 51:79-83.
Feinsinger, P. y R. K. Colwell. 1978. Community organization among neotropical nectar-feeding birds. American Zoologist 18:779-795.

Garrison, J. S. E. y C. L. Gass. 1999. Response of a traplining hummingbird to changes in nectar availability. Behavioral Ecology 10:714-725.

Gass, C. L. y R. D.Montgomerie. 1981. Hummingbird foraging behavior: decision making and energy regulation. In Foraging behavior: ecological, ethological, and psychological approaches, A. C. Kamil y T. D. Sargent (eds.). Garland, New York. p. 159-194.

Gass, C. L. y G. D. Sutherland. 1985. Specialization by territorial hummingbirds on experimentally enriched patches of flowers: energetic profitability and learning. Canadian Journal of Zoology 63:2125-2133.

Gass, C., M. Romich y R. Suárez. 1999. Energetics of hummingbird foraging at low ambient temperature. Canadian Journal of Zoology 77:314-320.

George, M. W. 1980. Hummingbird foraging behavior at Malvaviscus arboreus var. Drummondii. Auk 97:790-794.

González-Gómez, P. L. y R. A. Vásquez. 2006. A field study of spatial memory in Green backed firecrown hummingbirds (Sephanoides sephaniodes). Ethology 112:790-795.

Grant, V. y E. J. Temeles. 1992. Foraging ability of rufous hummingbirds on hummingbird flowers and hawkmoth flowers. Proceedings of the National Academy of Sciences of the United States of America 89:9400-9404.

Healy, S. D. y T. A. Hurly. 1995. Spatial memory in rufous hummingbirds (Selasphorus rufus): a field test. Animal Learning and Behaviour 23:63-68.

Healy, S. D. y T. A. Hurly. 1998. Rufous hummingbirds' (Selasphorus rufus) memory for flowers: patterns or actual spatial locations? Journal of Experimental Psychology: Animal Behavior Processes 24:396-404.

Healy, S. D. y T. A. Hurly. 2001. Foraging and spatial learning in hummingbirds. In Cognitive ecology of pollination: animal behavior and floral evolution, L. Chittka y J. D. Thomson (eds.). Cambridge University Press. p. 128-147.

Healy, S. D. y T. A. Hurly. 2003. Cognitive ecology: foraging in hummingbirds as a model system. Advances in the Study of Behavior 32:325-359.

Heinrich, B.1983. Do bumblebees forage optimally, and does it matter? American Zoologist 23:273-281.

Henderson, J., T. A. Hurly y S. D. Healy. 2001. Rufous hummingbirds' memory for flower location. Animal Behaviour 61:981-986.

Hurly, T. A. 1996. Spatial memory in rufous hummingbirds: memory for rewarded and non-rewarded sites. Animal Behaviour 51:177-183.

Hurly, T. A. y S. D. Healy. 1996. Memory for flowers in rufous hummingbirds: location or local visual cues? Animal Behaviour 51:1149-1157. 
Hurly, T. A. y S. D. Healy. 2002. Cue learning by rufous hummingbirds (Selasphorus rufus). Journal of Experimental Psychology: Animal Behavior Processes 28:20-23.

Irwin, R. 2000. Hummingbird avoidance of nectar-robbed plants: spatial location or visual cues. Oikos 91:499-506.

Lara, C. 2006. Temporal dynamics of flower use by hummingbirds in a temperate forest in México. Ecoscience 13:23-29.

Lara, C. y J. F. Ornelas. 2008. Pollination ecology of Penstemon roseus (Plantaginaceae), an endemic perennial shifted toward hummingbird specialization? Plant Systematics and Evolution 271:223-237.

Lara, C., K. Lumbreras y M. González. 2009. Niche partitioning among hummingbirds foraging on Penstemon roseus (Plantaginaceae) in central Mexico. Ornitologia Neotropical 20:73-83.

Melendez-Ackerman, E., D. R. Campbell y N. M. Waser. 1997. Hummingbird behavior and mechanisms of selection on flower color in Ipomopsis. Ecology 78:2532-2541.

Miller, R. S. y R. E. Miller.1971. Feeding activity and color preference of ruby-throated hummingbirds. Condor 73:309313.

Miller, R. S., S. Tamm, G. D. Sutherland y C. L. Gass. 1985. Cues for orientation in hummingbird foraging: color and position. Canadian Journal of Zoology 63:18-21.

Muenchow, G. 1986. Ecological use of failure time analysis.
Ecology 67:246-250.

Pyke, G. H. 1978a. Optimal foraging in hummingbirds: testing the marginal value theorem. American Zoologist 18:739-752.

Pyke, G. H. 1978b. Optimal foraging: movement patterns of bumblebees between inflorescences. Theoretical Population Biology 13:72-98.

Schuchmann, K. L. 1999. Family Trochilidae (Hummingbirds). In Handbook of the birds of the world, vol. 5., J. del Hoyo, A. Elliott y J. Sargatal (eds.). Lynx, Barcelona. p. 468-680.

Stiles, F. G. 1976. Taste preferences, color preferences, and flower choice in hummingbirds. Condor 78:10-26.

Sutherland, G. D. y C. L. Gass. 1995. Learning and remembering of spatial patterns by hummingbirds. Animal Behavior 50:1273-1286.

Sherry, D. F. 1985. Food storage by birds and mammals. Advances in the Study of Behavior 15: 153-188.

Williamson, S. L. 2001. A field guide to hummingbirds of North America. Peterson Field Guide Series. Houghton Mifflin, Boston, Massachusetts. 280 p.

Wolf, L. L. y F. R. Hainsworth. 1990. Non random foraging by hummingbirds: patterns of movement between Ipomopsis agregata (Pursch.) V. Grant inflorescences. Functional Ecology 4:149-157.

Zar, J. H.1999. Biostatistical analysis, cuarta edición. Prentice Hall, New Jersey. 662 p. 